\title{
The new generation magnetic-iron-detector to measure the iron overload in the human liver
}

\author{
Barbara Gianesin ${ }^{1,2,3^{*}}$, Alessio Caminata ${ }^{1,2}$, Piergiorgio Beruto ${ }^{2}$, Francesco Romoli ${ }^{1}$ and Mauro Marinelli ${ }^{1,2}$ \\ *Correspondence: gianesin@ge.infn.it \\ 'Department of Physics, University of Genoa, Italy. \\ ${ }^{2}$ National Institute of Nuclear Physics, Genoa, Italy. \\ ${ }^{3}$ E.O. Ospedali Galliera, Genoa, Italy.
}

\begin{abstract}
Background: The knowledge of liver iron overload is essential for the diagnosis and the therapy of diseases which induce systemic iron overload in the body. The liver needle biopsy is a well-known invasive method to measure liver iron concentration. The Magnetic Iron Detector (MID) susceptometer has proven to be a non-invasive apparatus capable of measuring the iron overload in the whole liver and also managing the follow-up therapy.

Methods: The iron overload is obtained by calculating the difference between the magnetization signal of the patient and his/her background signal, which is defined as the magnetization signal that the patient would generate with the basal iron content. The first order approximation of the background signal is calculated from a $3 \mathrm{D}$-laser-scan of the body of the patient. A calibration with healthy volunteers is needed to obtain a model for the calculation of the background signal.

Results and Conclusions: From 2005, the MID was used by the Galliera Hospital (Genoa, Italy) and about 2000 evaluations of iron overload were carried out with this instrument. A new generation of MID was developed to substitute the former susceptometer. The project for this new apparatus was started in 2009 and the new version was installed in February 2012 at Galliera Hospital where it is currently in use. The paper describes the new generation of MID and shows how it works focusing on the improvements regarding the engineering of the apparatus, the user interface and the measurement time. Moreover, we herein report the measurement performed on a phantom, the results of the calibration with 69 healthy volunteers and the description of the model by which we calculate the background signal.
\end{abstract}

Keywords: Liver iron overload, liver iron concentration, magnetic iron detector, susceptometry

\section{Introduction}

The knowledge of liver iron overload is essential for the diagnosis and therapy of diseases such as thalassemia or hemochromatosis, which induce systemic iron overload in the body [1]. The liver needle biopsy is a well-known method to measure liver iron concentration. This procedure is invasive and can lead to considerable errors due to the heterogeneous distribution of iron deposits in the liver [2]. Magnetic Resonance Imaging and biosusceptometry are technique capable to detect iron overload in the body [3-8]. By contrast with these techniques, which return the iron concentration, the Magnetic Iron Detector (MID) susceptometer has proven to be a non-invasive apparatus capable of measuring the iron overload in the whole liver and also managing the follow-up therapy (phlebotomy, chelation therapy) [9-11]. The MID measurements were previously compared with the results of biopsy, SQUID-Susceptometry and the $\mathrm{T}^{*}$ measurements obtained from Magnetic Resonance Imaging $[10,11]$.

The MID [9-12] quantifies the amount of iron in the liver measuring the human body's magnetic susceptibility. The magnetization induced in the human body is mainly due to tissue diamagnetism. It reduces the magnetic field surrounding the body by about one part in a million. In addition to this decreasing effect, there is also a smaller increment due to the paramagnetism of the iron atoms $[\mathbf{9}, \mathbf{1 0}]$. The MID determines iron overload by calculating the difference between the magnetization signal of the patient and his/her background signal, defined as the magnetization signal that the patient would generate with basal iron content. The evaluation of the background signal is performed under the hypothesis that the magnetization signal of a patient, with no iron overload, is the same as the one of a healthy volunteer with the same anthropometric characteristics.

The MID has been in use at the Galliera Hospital in Genoa, Italy, since February 2005 and has been used for more than 2000 iron overload examinations (Feb 2005-Feb 2012) [10]. In this paper, we describe the new generation of MID, which was developed to substitute the former susceptometer. The project was started in 2009 by way of a joint collaboration between the National Institute of Nuclear Physics (INFN), the "E. O. Ospedali Galliera" hospital and the non-profit patient association "Associazione Ligure Talassemici". The development, the construction and the relative tests were all performed at the INFN laboratories of Genoa. The new version of the susceptometer was installed in February 2012 and is currently in use at Galliera Hospital.

\section{Methods \\ Instrument description}

Two identical pickup coils are symmetrically assembled with respect to an electromagnet in order to cancel out their 


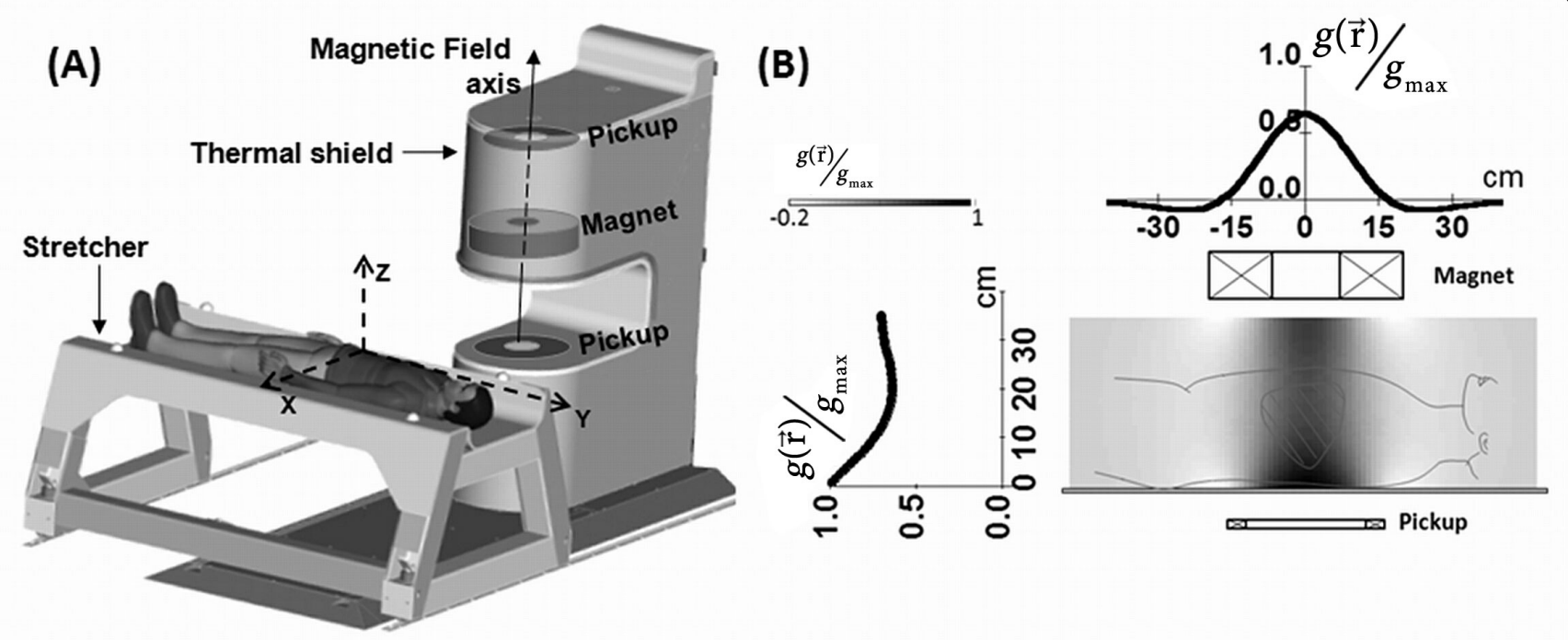

Figure 1. (A) A sketch of the MID susceptometer. The stretcher moves on rails along the X direction. (B) The spatial distribution of the weight-function, assuming cylindrical symmetry of the magnet and pickup coil system.

induced voltage when the apparatus is unloaded (Figure 1A). The electromagnet is energized by a sine-wave time dependent current and the magnetic field induces the same voltage in both pickup coils. The magnet has 192 turns with a mean diameter of $13 \mathrm{~cm}$ and the pickup coil is made by 800 turns with a mean diameter of $14.6 \mathrm{~cm}$. The magnet current has an amplitude of $50 \mathrm{~A}$ and a frequency of $144 \mathrm{~Hz}$. The peakvoltage induced across each pickup is $28 \mathrm{~V}$. When the patient is positioned between the magnet and the lower pickup, namely, the measurement region, the voltage induced in the lower pickup coil becomes different from the voltage induced in the upper pickup coil. This difference is the signal that is one part per million of the peak-voltage [9]. It is measured by the lock-in amplifier (EG\&G 7260), locked with the reference magnet-oscillating current. Figure $2 \mathrm{~A}$ shows the fiber-reinforced resin structure holding the magnet and the pickup coils. The symmetric arrangement of the magnet and pickup coils makes the difference between induced voltages less than $0.1 \mathrm{~V}$. After appropriate trimming of the number of turns of the pickup coils, this difference becomes less than $300 \mu \mathrm{V}$. A control system reduces this difference to less than $50 \mu \mathrm{V}$ by feeding appropriate current, phase-locked with the magnet current, in a small coil coupled with one of the pickup coils. The amplitude and phase of this current remains unchanged during the patient's measurement time.

The amplitude of the MID magnetic field applied to the human body has been calculated knowing the geometry of the magnet and has been measured by F. W. Bell gaussmeter model 615 . It has been also checked by means of a circular coil of 1000 turns and diameter of $18.4 \mathrm{~cm}$. The measured field conforms to the European Standard CEI EN 60601-2-33. The field amplitude is less than $1.9 \times 10^{-2} \mathrm{~T}$ within the entire measurement region; it is less than $9.0 \times 10^{-3} \mathrm{~T}$ at $30 \mathrm{~cm}$ over the stretcher on the magnetic field axis and it decreases to $2 \times 10^{-4} \mathrm{~T}$ at $30 \mathrm{~cm}$ from the MID external surface.

The main source of signal drift is the variation of the mutual inductance between the magnet and the pickup coils that is primarily caused by geometrical changes in the apparatus. To avoid this source of drift, the temperature of the entire susceptometer is kept steady within few millikelvin by controlling the temperature of the low conductivity water flowing through the hollow conductor of the magnet and through the channels embedded in all the walls and the roof of the thermal shield. The temperature is measured by a 7-1/2 digits multimeter (Keithley Instruments Inc. Model 2010) using a negative temperature coefficient (NTC) thermistors as transducer. The thermal shield (Figure 2B) covers the entire fiber-reinforced resin structure without actually touching it.

The distance between the pickup coils and the magnet is $0.45 \mathrm{~m}$ and the aperture of the thermal shield, suitable for the human body, is $0.36 \mathrm{~m}$. It should be noted that in the previous MID, this aperture was narrower $(0.27 \mathrm{~m})$ as it was originally designed to measure thalassemic patients. However, when patients with other pathologies started being measured, roughly $20 \%$ had to be excluded due to the restricted area of the opening. As a further improvement, the diameter of the magnet and pickup coils has been optimized to maximize the contribution to the magnetization signal by the liver.

The instrumentation is controlled by means of the National Instrument NI-PXI system and custom software developed in Labview The MID web-based user interface guides the entire patient workflow, from measurement to reporting. The user interface runs on a dedicated server to increase confidential data security and service availability. Any authorized MID user can access the database remotely to retrieve patient data history. 

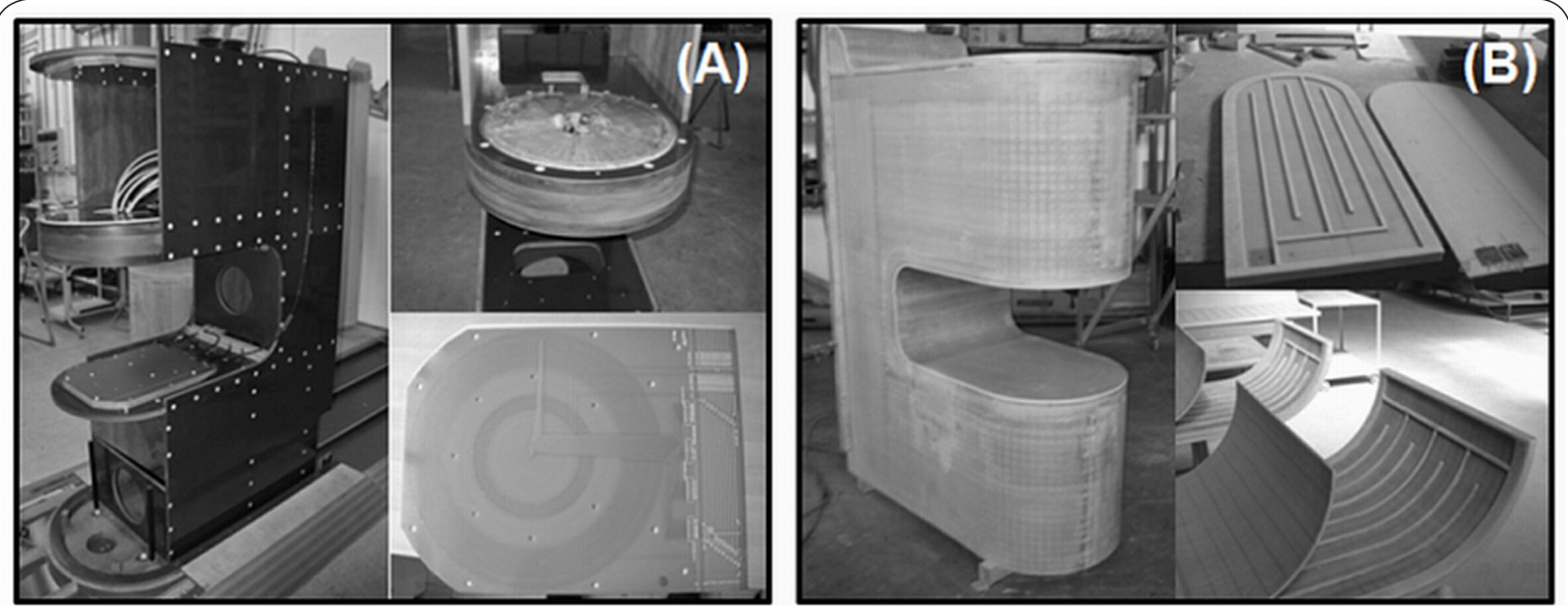

Figure 2. (A) A fiber-reinforced resin structure holds the magnet and pickup coils (left). The magnet and one pickup coil (right). The pickup coils are made by printed circuit board. (B) The assembled thermal shield and its components.

\section{Positioning the patient on the stretcher}

The patient is placed in the measurement region on a stretcher with small magnetic properties. The stretcher is made of a light honeycomb structure of electrical insulating fiber and it is able to handle a maximum weight of $600 \mathrm{~kg}$ to limit deformation to less than a few millimeters even under the load of bariatric patients. The coordinate system is fixed to the stretcher and the origin is located on the stretcher plane (Figure 1A). The XZ-plane contains the magnetic field axis and the $\mathrm{Y}$-axis is located at the center of the stretcher plane. The patient position on the stretcher is such that the $\mathrm{Y}$-axis, positively oriented towards the head, lies along the posterior mid-line of the body. The Y-coordinate of the liver center of mass is equal to zero and the $\mathrm{X}$-coordinate is negative and it is located between the coordinates $X=-4 \mathrm{~cm}$ and $X=-8 \mathrm{~cm}$ [10]. The physician finds the coordinate of the liver center of mass by percussion and no ultrasound devices are required. The magnetic scan of the body is performed by moving the stretcher along the rails in the $\mathrm{X}$ direction. An XY coordinate grid is projected onto the stretcher in order to allow for a more accurate positioning of the patient (Figure 3B).

\section{Measurement of the 3D-body shape}

Before each magnetic scan, a 3D-shape of the patient body is obtained by means of a laser scanner (Danish Sensor Engineering Aps model O2DS-1950, $2 \mathrm{kHz}$ scan frequency) mounted directly to the ceiling above the patient (Figure 3C). The O2DS-1950 records the $Y-Z$ coordinates of the body while the stretcher moves slowly along the $X$-axis. When the laser beam is parallel to the Z-axis, a trigger signal is generated and the position of the stretcher is recorded. In this way, it is possible to combine the $\mathrm{Y}-\mathrm{Z}$ laser measurement with its $\mathrm{X}$ coordinate.

Moreover, to prevent possible errors due to the misalignment of the laser system (eg., for thermal expansions), four semispherical markers are placed in the corners of the stretcher (Figure 3D). Knowing the position of the markers relative to the stretcher, a roto-translation is applied to the acquired data minimizing the distance between the acquired and the known positions of the markers. Typically, both the translation vector and the Euler angles of the rotation are negligible proving the stability of the apparatus. Figure $3 \mathbf{A}$ shows the laser system. It takes about 1 minute to acquire a full-body scan (Figure 3D). In order to obtain a correct measurement of the 3D-body shape, the patient wears only underwear whose magnetic properties have been previously measured. During the magnetic scan, the patient can be covered with a light sheet, should he/she wish.

\section{The magnetic scan}

The magnetic scan of the patient's body consists of 7 magnetization signals at different positions as stretcher enters the magnetic field region. Each magnetization signal is computed by averaging three differences between the measurements with the patient in and out of the magnetic field region and subtracting the magnetic signal of the stretcher from this average value. The magnetization signal of the stretcher is $(0.5 \pm 0.1) \mu \mathrm{V}$. Typically, the standard deviation of the 3 measured differences is less than $0.3 \mu \mathrm{V}$. For comparison, as discussed in section 3 below, the magnetic signal of one gram of ferritinhemosiderin iron evenly distributed in the liver is about $1.2 \mu \mathrm{V}$. In cases when the standard deviation of the three measured differences is larger than $0.3 \mu \mathrm{V}$, we repeat the magnetization signal measurement and compute the weighted average of the two results. This procedure is repeated until the standard deviation is less than $0.3 \mu \mathrm{V}$. This happens for roughly $7 \%$ of the exams during regular hospital activities while the number of repeated measurements becomes negligible during low 


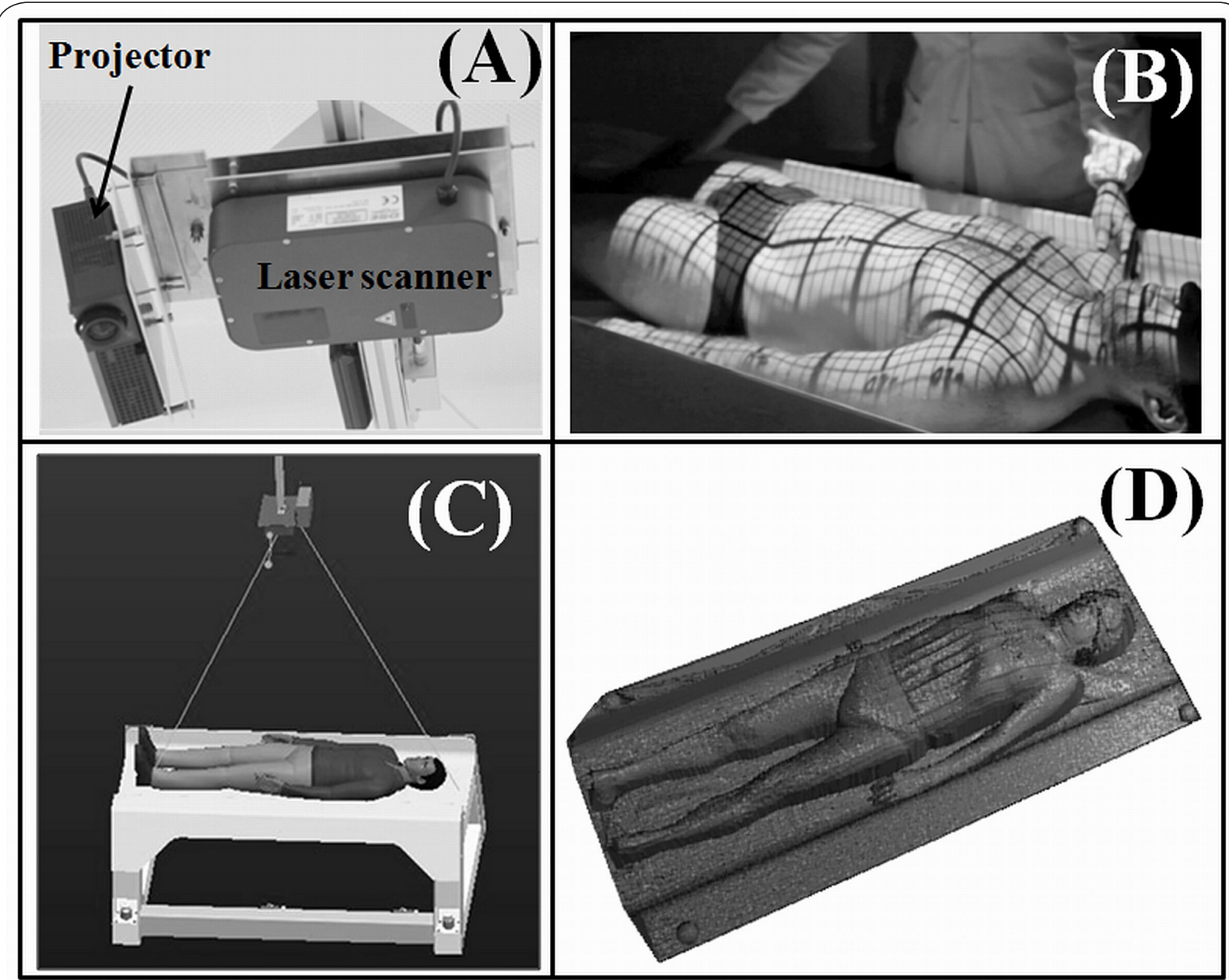

Figure 3. (A) Laser scanner DSE 1950 and XY grid projector. (B) The XY coordinate grid projected onto the stretcher. (C) Sketch of the laser-stretcher system. The laser scanner is mounted directly to the ceiling above the patient. The stretcher moves along the rails during the data collection. (D) Example of the 3D-body shape (male volunteer).

hospital activity (eg., week-ends). Being the variability of the magnetization signal, due to mechanical and electrical changes of the apparatus, negligible, it is inferred that the dominant source of variability in the magnetic measurements is due to variations in background magnetic properties (eg., change in position of ferromagnetic objects in nearby hospital rooms). The MID takes roughly 17 minutes to acquire these 7 measurements and begins this task after detecting the 3D-body shape.

\section{The MID signal}

A human body, with a distribution of magnetic susceptibility $\chi(\vec{r})$ and a volume $V$, placed between the magnet and the pickup coil, generates a magnetization signal as follows:

$$
S(X)=\int_{V} g(\vec{r}-X \vec{i}) \chi(\vec{r}) d \vec{r}
$$

where the vector $\vec{r}$ runs from origin of the XYZ coordinate system to the elementary volume $d \vec{r}$ within the body, $X$ is the coordinate of magnetic field axis in the XYZ coordinate system and $\vec{i}$ is the $\mathrm{X}$-axis unit vector. The weight function accounts for the contribution to the magnetization signal of a unit volume of matter with unitary magnetic susceptibility. The magnitude of $g(\vec{r})$ depends on the frequency and amplitude of the current flowing in the magnet and on the magnet and pickup coil position and dimensions and it is calculated using the multi-physics engineering simulation software ANSYS assuming cylindrical symmetry. The peak value of $g(\vec{r})$ is $1390 \mathrm{~V} / \mathrm{m}^{3}$.

The apparatus sensitivity and reproducibility was validated by measuring several times the magnetic properties of a phantom. The measured magnetization signal was compared to the one obtained from (1), given the geometry and magnetic properties of the phantom. The comparison was then performed in several $X$ positions. The phantom is a plastic container filled with 5.6 liter of low conductivity water $(10-20 \mathrm{k} \Omega \mathrm{cm})$. It has a cylindrical shape with a $0.200 \mathrm{~m}$ diameter of and a $0.177 \mathrm{~m}$ height. The phantom was placed 


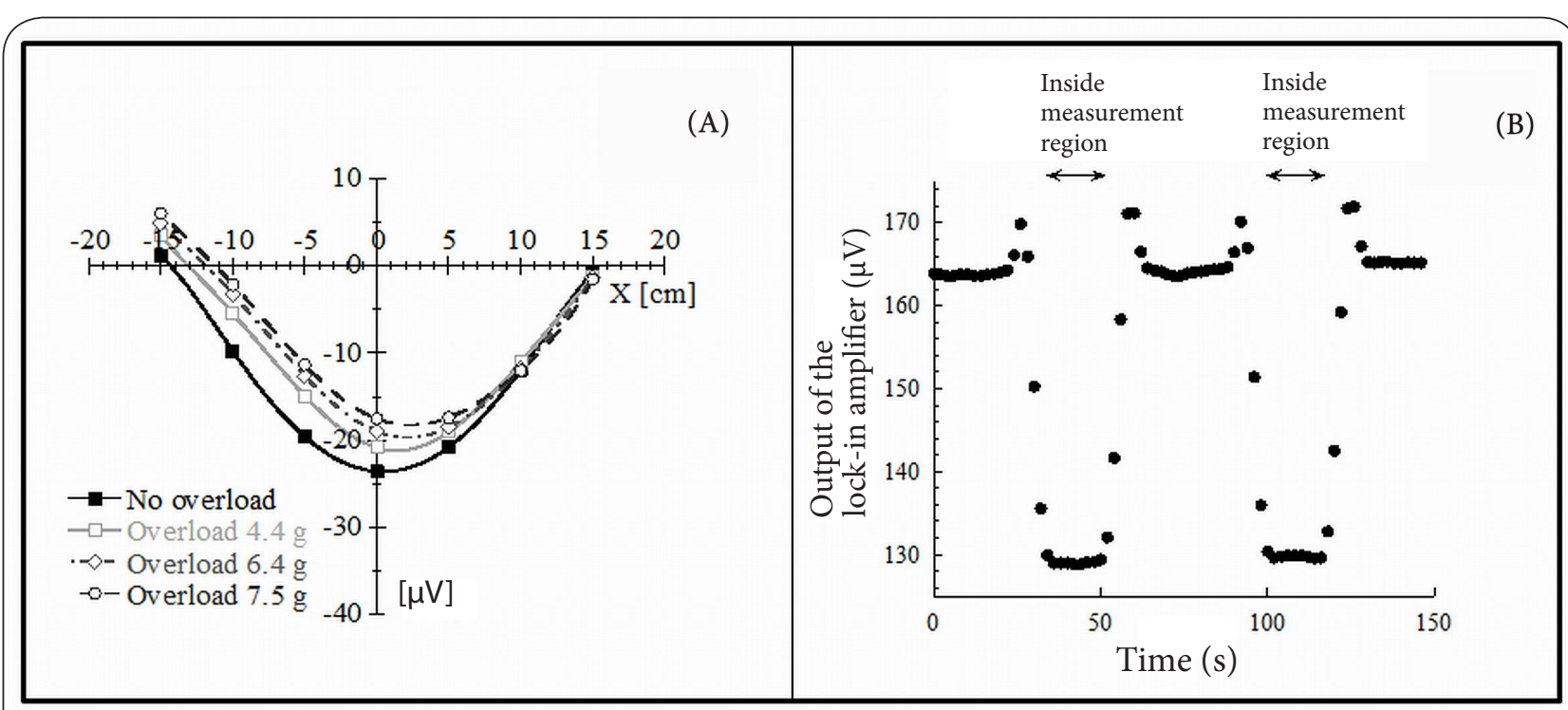

Figure 4. (A) Magnetization signals of the anthropomorphic phantom. (B) Output of the lock-in amplifier during a magnetic scan of the human body.

on the stretcher with its axis crossing the origin of the $\mathrm{XYZ}$ coordinate system (Figure 1A).

The oscillating magnetic field induces also eddy currents in the human body that generate a signal in addition to the magnetization one. The phase difference between magnetization and eddy current signals at the frequency of our oscillating field is 90 degrees and thus, as for the first-generation MID, the two components of the signal are discriminated via phase-locked detection with a lock-in amplifier $[\mathbf{9 , 1 0}]$.

\section{Background signal calculation}

The background signal is defined as the signal generated by the human body with the basal iron in the liver. The amount of iron overload is proportional to the difference between the magnetization signal and the background signal $[\mathbf{9 , 1 0 ]}$. Therefore, it is necessary to evaluate the latter by means of a model. The first step to evaluate the background signal is the calculation in (1), with the integration volume bounded by the body in the 3D-body shape and assuming a uniform magnetic susceptibility equal to that of water: this is the waterman. Because the 3D-body shape (Figure 3D) contains both the shape of the patient and of the stretcher during the calculation, the contribution of the stretcher is subtracted using a previously acquired shape of the plain stretcher.

The waterman overestimates the magnitude of the background signal because:

a) The volume bounded by the 3D-body shape is larger than the actual body volume as the laser is unable to account for empty space between the body and the stretcher.

b) The absolute value of the magnetic susceptibility of certain regions of the body (eg., the lungs) is less than that of water.
To decrease the void space between the stretcher and the back, before the positioning, we ask the patient to adduce the knees towards the chest to flattening his/her back. The residual void volume is estimated to be about one liter over the whole trunk. The shape of $g(\vec{r})$ ensures that only a part of this void space contributes to the waterman for every $\mathrm{X}$-position.

To evaluate the systematic error due to the residual void spaces and the difference in magnetic susceptibility, we compare the magnetization signal and the waterman of healthy volunteers. We measured the differences between the magnetization signal and the waterman, in a group of healthy volunteers, at $7 \mathrm{X}$-positions of the magnetic field axis relative to the center of the torso of the volunteers, and averaged these differences (table embedded in Figure 5).

The background signal of any new subject is then obtained by calculating the waterman in the $7 \mathrm{X}$-positions and adding these 7 mean differences. The same method was also used to calculate the background signal for the previous MID measurements [10].

\section{Human subject}

The calibration dataset consists of 69 healthy volunteers: 42 (61\%) male, 27 (39\%) female, age 23-78 (mean 42, STD=16), height 1.53-1.84 m (mean $1.71 \mathrm{~m}, \mathrm{STD}=0.08 \mathrm{~m}$ ) and weight $45-101 \mathrm{~kg}$ (mean $70 \mathrm{~kg}, \mathrm{STD}=13 \mathrm{~kg}$ ). All volunteers provided prior written informed consent.

\section{Errors in the measurement of iron overload}

The iron overload error depends on the measurement error of the magnetization signal as well as the error in the calculation of the background signal.

The instrumental error (i.e., the measurement error of 
Gianesin et al. Medical Instrumentation 2013,

\begin{tabular}{|ccc|}
\hline $\mathbf{X}$ & $<\mathbf{S}_{\mathbf{m}}-\mathbf{S}_{\mathbf{w}}>$ & $\begin{array}{c}\boldsymbol{\sigma}_{\mathbf{S m}-\mathbf{S b}} \\
\mathbf{\mu} \mathbf{V}\end{array}$ \\
\hline-15 & 3.7 & 1.5 \\
-10 & 5.3 & 1.2 \\
-5 & 5.5 & 1.3 \\
0 & 5.1 & 1.6 \\
5 & 5.3 & 1.4 \\
10 & 5.3 & 1.4 \\
15 & 3.5 & 1.6 \\
\hline
\end{tabular}

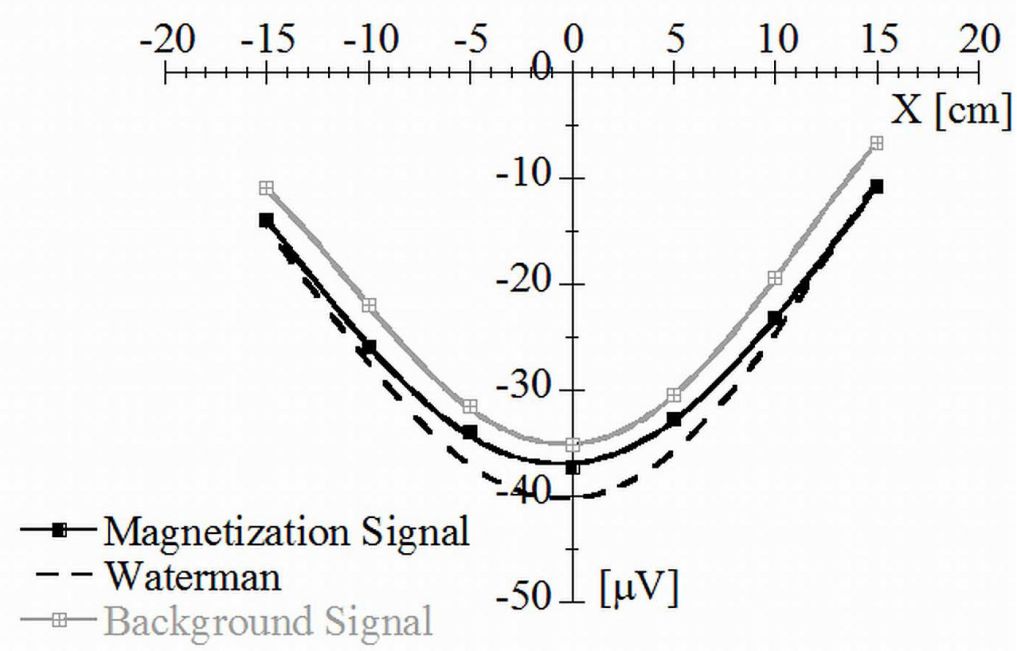

Figure 5. Magnetization signal, waterman and background signal of the volunteer in Figure 3D. The table reports the mean of the distribution of the differences between the magnetization signal Sm and the waterman Sw of volunteers and the standard deviation of the distribution of the differences.

the magnetization signal of a phantom on the stretcher) and the re-positioning error of the body on the stretcher contribute to the measurement error of the magnetization signal. The instrumental error was evaluated by measuring repeatedly the signal of the cylindrical phantom, which is centered at the origin of the XYZ coordinate system, and calculating the standard deviation of the distribution of the measurements. The re-positioning error was evaluated by positioning a volunteer on the stretcher several times and measuring each time the magnetization signal in $X=-5 \mathrm{~cm}$ position. After each measurement, the volunteer was asked to get off and back on the stretcher to be re-positioned. The standard deviation of the distribution of these magnetization signals takes into account both the instrumental error and the re-positioning error.

From (1) the body-volume measurement error, the uncertainty in the function $g(\vec{r})$ and the distribution of the susceptibility $\chi(\vec{r})$ can be identified as source of error in calculating the background signal. To verify the measurement error of the laser-stretcher system we measured the volume of a cylindrical phantom $(5600 \mathrm{cc})$ with an uncertainty of $80 \mathrm{cc}$. The volume is obtained calculating the waterman, using the acquired 3D-shape and assuming $g(\vec{r})=1$ and $\chi(\vec{r})=1$. The phantom was placed on the stretcher in the position taken up by the liver region. The error of the $\mathrm{X}$-coordinate measurements is about one millimeter, which is the uncertainty on the position of the stretcher. The error on the Z-coordinate is less than one millimeter. The main source of error is due to the spatial resolution of the laser measurements along Y. Because of the cam mechanism of the laser system, the error on the Y-direction varies with the beam orientation but it is less than $5 \mathrm{~mm}$, which is the maximum distance between two consecutive acquired points.
Concerning the function $g(\vec{r})$, its actual value differs from the theoretical value by less than $10 \%$. This estimate was obtained by measuring the magnetization signal of the cylindrical phantom, positioned at first with its axis aligned with the magnetic field axis, then $20 \mathrm{~cm}$ and $40 \mathrm{~cm}$ off axis and finally comparing the measurements with the results of the calculation in (1). Summing-up, the difference between the magnetization signal of a healthy volunteer and his/ her waterman depends on the non-uniform distribution of the magnetic susceptibility $\chi(\vec{r})$ within the patient body, the volume bounded by the 3D-body shape and the difference between the actual value of the function $g(\vec{r})$ and its theoretical value. The procedure of calculation of the background signal corrects the systematic errors coming from these contributions.

\section{Results}

The distribution of the magnetization signals of the cylindrical phantom, positioned as described in substitute with "the previous section" has an average value of $-30 \mu \mathrm{V}$ and a standard deviation of $0.2 \mu \mathrm{V}$, which is the instrumental error of the MID. A final check for the instrumental error is performed using an anthropomorphic phantom (RSD, Alderson Rando Therapy phantom). The magnetic susceptibility of the phantom has been changed spreading, within its hepatic region, different quantities of paramagnetic sand whose signals are equivalent to $4.4 \mathrm{~g}, 6.4 \mathrm{~g}$ and $7.5 \mathrm{~g}$ of ferritin-haemosiderin iron. The relative magnetization signals are shown in Figure 4A. The equivalent iron overload sensitivity of these measurements is about $0.4 \mathrm{~g}$.

As for the reproducibility of a human body re-positioning, the standard deviations of the magnetization signal measurements and the waterman calculations are $0.5 \mu \mathrm{V}$. All the 
watermen have been calculated in the $X=-5 \mathrm{~cm}$ position, using the 3-D body shape acquired before each magnetization signal measurement.

The standard deviation of the distribution of the magnetization signals, acquired without any movement of the volunteer on the stretcher, is roughly $0.3 \mu \mathrm{V}$. The lock-in amplifier (time constant $2 \mathrm{sec}, 12 \mathrm{~dB} / \mathrm{oct}$ ) output, with the patient inside the measurement region, does not show a synchronous change with the patient's breath (Figure 4B). This is due to the smooth variation of the weight function $g(\vec{r})$ along the $Z$ direction (Figure 1B). The typical magnetization signal of a volunteer in such a position is $-35 \mu \mathrm{V}$.

The signal of a gram of ferritin-hemosiderin iron, evenly distributed in the liver, was calculated by (1) for several liver volumes. The position of the liver relative to the magnetic field is such that the patient lies on the stretcher with the center of mass of the liver on the magnetic field axis. As a reference, $1 \mathrm{gram}$ of iron evenly distributed in a liver of 1300 cc generates a signal of $+1.2 \mu \mathrm{V}$.

The table embedded in Figure 5 shows the mean of the differences between the magnetization signal and the waterman $\left(\left\langle S_{m}-S_{w}\right\rangle\right)$ of 68 volunteers in the $7 \mathrm{X}$-positions and the standard deviations $\left(\sigma_{\mathrm{Sm}-\mathrm{Sb}}\right)$ of the distribution of the differences between the measured magnetization signal and the calculated background signal. One volunteer was excluded from the original dataset being the difference $\left\langle\mathrm{S}_{\mathrm{m}}-\mathrm{S}_{\mathrm{w}}\right\rangle$ greater than 3 standard deviations; the "leave-one-out" cross validation technique was applied to the volunteer data set. The plot in Figure 5 shows the magnetization signal, the waterman and the background signal of one male volunteer, whose 3D-body shape is shown in Figure 3D; the background signal was obtained by adding the mean of the differences of the other 67 volunteers to his waterman.

The evaluation of the liver iron overload comes from the difference between the magnetization signal and the background signal with the liver center of mass on the magnetic field axis, i.e., $X$ between $-5 \mathrm{~cm}$ and $-10 \mathrm{~cm}$. The present sensitivity of the iron overload measurement is about $1.1 \mathrm{~g}$ which is the ratio between the highest value of $\sigma_{\mathrm{Sm}-\mathrm{Sb}}$ in this region $(1.3 \mu \mathrm{V})$ and the signal per gram of iron evenly distributed in the liver $(1.2 \mu \mathrm{V} / \mathrm{g})$.

\section{Discussion}

The sensitivity of the MID is currently limited by the calculation of the background signal. In fact, all the standard deviations reported in the table embedded in Figure 5 are substantially greater than both the measurement error of the magnetization signal of the body and the error in the evaluation of the waterman. Our plan is to develop models to calculate the background signal for different classes of patients (eg., male/ female, body mass index). Moreover, with a larger number of volunteers, it is also possible to develop new models for estimating the background signal from the waterman, studying, for example, the dependence of the correction on the anthropometric characteristics of the patient (eg., the width of the chest or the height).

Thanks to a wider measurement region (from $0.27 \mathrm{~m}$ to $0.36 \mathrm{~m}$ ), the current MID can measure liver iron overload in a broader patient population, enabling measurements of bariatric patients. In spite of the greater distance between the magnet and pickup coil, the signal-to-noise ratio is the same or slightly higher than the one of the previous MID. The magnetization signal of the volunteer in Figure $3 \mathrm{D}$ is $(37.3 \pm 0.5) \mu \mathrm{V}$. The magnetization signal of the same volunteer with the previous version of the susceptometer was $(4.7 \pm 0.1) \mu \mathrm{V}$. Both measurement time and user interface were also improved in the current MID. For example, patient positioning was simplified by the introduction of the projected XY-coordinate grid on the body and the time required to acquire the 3D-body shape was significantly reduced (from 12 minutes to 1 minute). In addition, workflow was streamlined; measurements require minimal interaction with the user and are easily managed by hospital personnel.

\section{Conclusions}

In this paper, we have presented the operation of the new generation MID. In addition to performance improvements, we have addressed previous ergonomics and workflow limitations. The current MID can serve a broader patient population, including bariatric patients, and its operation is faster and more automated. We installed the MID at Galliera Hospital in February 2012 and, since then, it has been in continuous operation.

\section{Competing interests}

The authors declare that they have no competing interests.

\section{Authors' contributions}

B. Gianesin was involved in the iron overload measurement project from its beginning. In particular, for the present MID, she used the acquired experience to collaborating to the design decisions. She worked also on the software to manage MID instrumentation and control and for the background signal model. A. Caminata chose to write up his $\mathrm{PhD}$ thesis on the iron overload measurement, consequently collaborating to the design decisions about this new MID version and on the software for the MID control and for the background signal calculation. P. Beruto designed the software for the construction of the 3D-body shape and for the control of the stretcher position. F. Romoli chose to write up his Master thesis on the iron overload measurement, consequently collaborating to the model and the test of the background signal calculation. M. Marinelli was the principal investigator. All the authors gave their contribution on writing the paper.

\section{Acknowledgement and funding}

The authors would like to thank "Associazione Ligure Talassemici" and INFN for funding this project. Dr G. L. Forni (Centre of Hematology and Microcitemia, Galleria Hospital) for the trust extended to us and for constructive discussions. The heads administration of INFN in Rome and Genoa, L. De Donno and S.Squarcia, for their contributions to the agreement with the "E.O. Ospedali Galliera" and ALT, and to M. Pavan for handling the ALT funding. Our acknowledgements is extended to the staff 
Gianesin et al. Medical Instrumentation 2013,

of the INFN: R. Cereseto designed the MID support structure, thermal screen and stretcher, and followed their construction; $M$. Cresta designed and put together the hardware to control the MID temperature, the stretcher movement, and all the electrical circuits; G. Sobrero contributed appreciated ideas to the design of the MID components and was involved in the cooling system; F. Pratolongo drafted all printed circuit boards and wrote the MID documentation and risk analysis; M. Parodi and C. Pizzorno for all their help and ideas during the assembly stage; R. Puppo for the final drawings of all the mechanical components; G. Gariano and A. Rovani for assembling some instrumentation for the temperature control; P. Pozzo for handling all shipping and deliveries. E. Carusi for the design of the user interface; M. Guideri and G. Parisi for having assisted in the planning stage of the magnet and pickup coils; A. Chincarini for his help in using Comsol and Matlab; C. Bruzzone for her collaboration in the drawing up of the software to manage the MID; the Information Technology staff of the INFN and of the Galliera Hospital, F. Saffioti and M. De Benedetto.

\section{Publication history}

Editor: Ioannis G. Valais, Technological Educational Institute, Greece.

EIC: Robert A. Lodder, University of Kentucky, USA.

Received: 07-Jun-2013 Revised: 27-Jun-2013

Accepted: 27-Jun-2013 Published: 13-Jul-2013

\section{References}

1. Angelucci E, Brittenham GM, McLaren CE, Ripalti M, Baronciani D, Giardini C, Galimberti M, Polchi P and Lucarelli G: Hepatic iron concentration and total body iron stores in thalassemia major. $N$ Engl J Med 2000, 343:327-31. | Article | PubMed

2. Butensky E, Fischer R, Hudes M, Schumacher L, Williams R, Moyer TP, Vichinsky $E$ and Harmatz $P$ : Variability in hepatic iron concentration in percutaneous needle biopsy specimens from patients with transfusional hemosiderosis. Am J Clin Pathol 2005, 123:146-52. | Article | PubMed

3. St Pierre TG, Clark PR, Chua-anusorn W, Fleming AJ, Jeffrey GP, Olynyk JK, Pootrakul $P$, Robins $E$ and Lindeman R: Noninvasive measurement and imaging of liver iron concentrations using proton magnetic resonance. Blood 2005, 105:855-61. | Article | PubMed

4. Wood JC, Enriquez C, Ghugre N, Tyzka JM, Carson S, Nelson MD and Coates TD: MRI R2 and R2* mapping accurately estimates hepatic iron concentration in transfusion-dependent thalassemia and sickle cell disease patients. Blood 2005, 106:1460-5. | Article | PubMed Abstract | PubMed Full Text

5. Brittenham GM and Badman DG: Noninvasive measurement of iron: report of an NIDDK workshop. Blood 2003, 101:15-9. | Article | PubMed

6. Brittenham GM, Farrell DE, Harris JW, Feldman ES, Danish EH, Muir WA, Tripp JH and Bellon EM: Magnetic-susceptibility measurement of human iron stores. N Engl J Med 1982, 307:1671-5. | Article | PubMed

7. Avrin WF and Kumar S: Noninvasive liver-iron measurements with a room-temperature susceptometer. Physiol Meas 2007, 28:349-61. | Article | PubMed Abstract | PubMed Full Text

8. Maliken BD, Avrin WF, Nelson JE, Mooney J, Kumar S and Kowdley KV: Room-temperature susceptometry predicts biopsy-determined hepatic iron in patients with elevated serum ferritin. Ann Hepatol 2012, 11:7784. | PubMed Abstract | PubMed Full Text

9. Marinelli M, Gianesin B, Avignolo C, Minganti V and Parodi S: Iron overload detection in rats by means of a susceptometer operating at room temperature. Phys Med Biol 2008, 53:6849-60. | Article | PubMed

10. Marinelli M, Gianesin B, Balocco M, Beruto P, Bruzzone C, Carrara $P$, Gallusi P, Macco A, Musso M, Oliveri E, Pelucchi S, Sobrero G, Villa R and Forni GL: Total iron-overload measurement in the human liver region by the magnetic iron detector. IEEE Trans Biomed Eng 2010, 57:2295303. | Article | PubMed
11. Gianesin B, Zefiro D, Musso M, Rosa A, Bruzzone C, Balocco M, Carrara P, Bacigalupo L, Banderali S, Rollandi GA, Gambaro M, Marinelli M and Forni GL: Measurement of liver iron overload: noninvasive calibration of MRI-R2* by magnetic iron detector susceptometer. Magn Reson Med 2012, 67:1782-6. | Article | PubMed

12. Marinelli M, Gianesin B, Avignolo C, Minganti V and Parodi S: Iron overload detection in rats by means of a susceptometer operating at room temperature. Phys Med Biol 2008, 53:6849-60. | Article | PubMed

\section{Citation:}

Gianesin B, Caminata A, Beruto P, Romoli F and Marinelli $M$. The new generation magnetic-irondetector to measure the iron overload in the human liver. Med Instrum. 2013; 1:5. http://dx.doi.org/10.7243/2052-6962-1-5 